\title{
Alpha-hemolysin nanopore allows discrimination of the microcystins variants $\uparrow$
}

Cite this: RSC Adv., 2019, 9, 14683

\author{
Janilson J. S. Júnior, ${ }^{\text {ad }}$ Thereza A. Soares, (D) ${ }^{\text {b }}$ Laércio Pol-Fachin, ${ }^{\text {bc }}$ \\ Dijanah C. Machado, ${ }^{a}$ Victor H. Rusu, ${ }^{\text {b Juliana P. Aguiar }}{ }^{\text {ad }}$ \\ and Cláudio G. Rodrigues (D)*ad
}

Microcystins (MCs) are a class of cyclic heptapeptides with more than 100 variants produced by cyanobacteria present in surface waters. MCs are potent hepatotoxic agents responsible for fatal poisoning in animals and humans. Several techniques are employed in the detection of MCs, however, there is a shortage of methods capable of discriminating variants of MCs. In this work we demonstrate that the $\alpha$-hemolysin $(\alpha \mathrm{HL})$ nanopore can detect and discriminate the variants (LR, YR and RR) of MCs in aqueous solution. The discrimination process is based on the analysis of the residence times of each variant of MCs within the unitary nanopore, as well as, on the amplitudes of the blockages in the ionic current flowing through it. Simulations of molecular dynamics and calculation of the electrostatic potential revealed that the variants of MCs present different charge distribution and correlated with the three patterns on the amplitudes of the blockages in the ionic current. Additionally, molecular docking

Received 18th December 2018 Accepted 1st April 2019

DOI: $10.1039 / \mathrm{c} 8 \mathrm{ra10384d}$

rsc.li/rsc-advances analysis indicates different patterns of interaction of the variants of MCs with two specific regions of the nanopore. We conclude that $\alpha \mathrm{HL}$ nanopore can discriminate variants of microcystins by a mechanism based mainly on electrostatic interaction. Finally, we propose the use of nanopore-based technology as a promising method for analyzing microcystins in aqueous solutions.

\section{Introduction}

Microcystins (MCs) are constituted by cyclic heptapeptides containing several non-proteinogenic amino acids, and represent a class of exotoxins produced by cyanobacteria species commonly present in surface waters of tropical regions in the northern and southern hemispheres. ${ }^{\mathbf{1 , 2}}$ MCs general sequence is cyclo-(D-Ala1X2-D-MeAsp3-Z4-Adda5-D-Glu6-Mdha7) where the numbers indicate the position of a given amino acid in the sequence (Fig. 1). The modified residues MeAsp (Masp), Adda and Mdha corresponds to D-erythro-beta methylisoaspartic acid, $(2 S, 3 S, 8 S, 9 S)$-3-amino-9-methoxy-2,6,8-trimethyl-10-phenyldeca$(4 E, 6 E)$-dienoic acid and methyldehydroalanine, respectively. Positions $\mathrm{X} 2$ and $\mathrm{Z} 4$ are occupied by variable L-amino acids,

${ }^{a}$ Departamento de Biofisica e Radiobiologia, Universidade Federal de Pernambuco, Avenida Prof. Moraes Rego, $s / n$, Cidade Universitária, Recife, Pernambuco, 50670-901, Brazil. E-mail: cgrufpe@gmail.com; Fax: +55 812126 8560; Tel: +55 81 21268535

${ }^{b}$ Departamento de Quimica Fundamental, Universidade Federal de Pernambuco, Cidade Universitária 50740-560, Recife, PE, Brazil

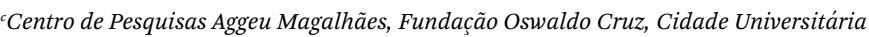
50740-465, Recife, PE, Brazil

${ }^{d}$ Programa de Pós-graduação em Inovação Terapêutica, Universidade Federal de Pernambuco, Brazil

$\dagger$ The authors dedicate this work to Dr Oleg Vladimirovich Krasilnikov (in memoriam). resulting in variants with different toxicity profiles. ${ }^{3}$ Currently over 100 variants of MCs have been described which differ from each other with respect to the identity of the two variable residues and chemical modifications in the other amino acids. ${ }^{4}$ The variant Microcystin-LR (MC-LR, $995 \mathrm{Da}$ ) containing the residues Leu2 and Arg4 is the most common and toxic of MCs., ${ }^{\mathbf{1 , 4}}$

MCs are potent hepatotoxic and nephrotoxic agents, which have been associated to liver, digestive and skin diseases, neurological impairment and death. ${ }^{5}$ It has been associated with most of the poisoning incidents worldwide. ${ }^{6}$ In February 1996, one of the largest toxic diseases caused by MCs in humans occurred in Caruaru in the Northeast of Brazil, resulting in the deaths of 60 patients. $^{7}$ MCs exert their toxicity via irreversible

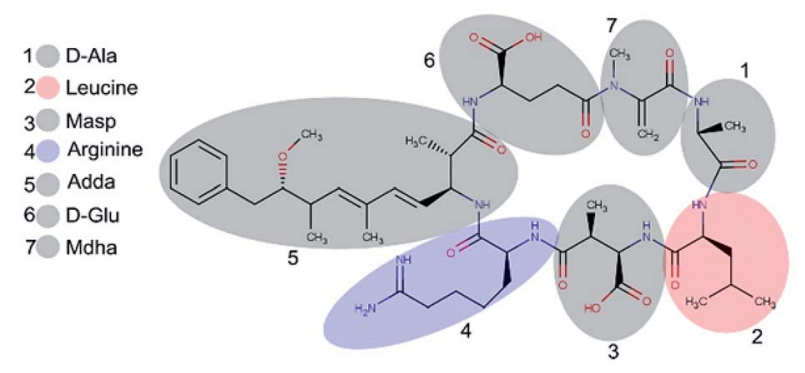

Fig. 1 Structure of microcystin-LR. Variants differ with respect to the identity of L-amino acids in position 2 and 4 . Numbers represent the position of the amino acid residue. 
inhibition of protein phosphatases 1 and 2A, affecting mainly liver cells, which can lead to death. ${ }^{8}$ Additionally, long-term exposure to low concentrations of MCs has been implicated in tumour growth promotion. ${ }^{9}$ The World Health Organization (WHO) recommends an upper limit of $1 \mu \mathrm{g} \mathrm{L}^{-1}$ of MCs in drinking water for human health protection. ${ }^{10}$ Eutrophication and climatic changes has led to the rapid proliferation and dominance of harmful blooms of cyanobacteria in freshwater and marine ecosystems. ${ }^{\mathbf{1 1}, 12}$ Temperatures ranging between 15$30{ }^{\circ} \mathrm{C}$ can selective promote cyanobacterial proliferation as the growth rates of these bacteria are optimal at relatively high temperatures. ${ }^{13}$ MCs are a growing threat to the use of freshwater ecosystems and reservoirs for drinking water, irrigation, and fishing. Therefore, fish and water consumption can be an important route of microcystin exposure for humans. ${ }^{\mathbf{1 4}}$

The low concentration and large number of variants represent a considerable challenge for the monitoring of MCs in water bodies, to which several analytical methods have been employed..$^{15}$ Among the most sensitive techniques are high performance liquid chromatography coupled with mass spectrometry (HPLC-MS), enzyme-linked immunosorbent assay (ELISA) and phosphatase assay. Some of these techniques offer excellent sensitivity and specificity but at high cost, timeconsuming sample preparation procedures and lengthy analysis. ${ }^{16}$ Furthermore, the use of HPLC-MS for the detection and identification of unknown MCs in environmental samples is hampered mainly by the lack of analytical standards for many MC variants. ${ }^{3,16}$ In addition, HPLC-MS requires highly skilled personal and expensive equipment. Protein phosphatase inhibition assays are susceptible to high rates of false-positive results, and cannot distinguish between different MC variants. ${ }^{3,17}$ Likewise, immunoassay techniques may present cross reactivity to MCs variants. ${ }^{17}$ Therefore, there is a critical demand for the development of low-cost analytical devices for MC monitoring in water, which features high sensitivity and specificity, easiness to use and great portability. Nanopore basedsystems have been successfully employed in the stochastic biosensing ${ }^{18,19}$ Stochastic biosensing is an approach that relies on the observation of individual binding events between analyte molecules and a single bioreceptor. ${ }^{20}$ Recently, solid-state nanopore with a diameter of approximately $10 \mathrm{~nm}$ was used to detect MC-LR via aptamer-conjugated with gold nanoparticles. ${ }^{21}$ In here, we used $\alpha$-hemolysin ( $\alpha \mathrm{HL}$ ) nanopore with diameter approximately $1 \mathrm{~nm}$ to discrimination microcystins variants. ${ }^{22}$ Nanopore formed by Staphylococcus aureus $\alpha$-hemolysin $(\alpha \mathrm{HL})$ is the most commonly protein nanopore employed in the stochastic biosensing technology. ${ }^{23}$ Because these pores are so small, their interaction with an analyte can alter the ionic current that would otherwise flow freely. This nanopore can be used as analytical tools for a wide variety of analytes (e.g. mass spectrometry of polymers, ${ }^{24}$ DNA sequencing, ${ }^{25}$ detection of enantiomers, ${ }^{26}$ cocaine $^{27}$ and poly(ethylene glycol)). ${ }^{28}$ In this work we study the interaction of MCs with the $\alpha$ HL-nanopore, aiming to develop a system for the detection and characterization of these cyanotoxins in aqueous systems. The choice of variants (LR, RR, YR) is due to higher occurrence in environmental water and also higher toxicity.

\section{Results and discussion}

$\alpha \mathrm{HL}$ nanopores exhibited uniform single-channel conductance state of long duration at both negative and positive transmembrane potentials. ${ }^{20}$ In $40 \mathrm{mV}$ an unobstructed $\alpha \mathrm{HL}$ nanopore has a constant current of approximately $150 \mathrm{pA}$ and conductance of $\sim 4 \mathrm{nS}$ in $\mathrm{KCl} 4 \mathrm{M}, \mathrm{pH} 7.5$, and the values of these parameters are in agreement with several previous reports. ${ }^{\mathbf{2 4 , 2 9 , 3 0}}$ The addition of MC-LR, MC-RR or MC-YR to the trans side of nanopore induces reversible partial blockades of the ionic current, and similarly to studies with other analytes, this clearly indicates that this nanopore is capable of detecting MCs, so it works as a molecular counter ${ }^{31-33}$ (Fig. 2).

The profile of the microcystin-induced ionic current blockages differ considerably from those reported in other studies using synthetic linear peptides with a number of amino acids similar to microcystins, suggesting that the nanopore is capable of discriminating the analytes by the molecular configuration. ${ }^{34,35}$ Although the MCs variants differ in only a single amino acid (Fig. 2A), each variants exhibits a typical characteristic blockages of the ionic current (Fig. 2B). Observe that the amplitude of the blockages of the ionic current caused by MCYR is larger compared to the other variants (Table 1, right column). The MC-YR has blocked about $\sim 91.6 \%$ of the ionic current, followed by $\sim 81.6 \%$ of the MC-RR and $\sim 70.1 \%$ of MCLR, respectively. Then, the magnitude of the ionic current blockages amplitude in the nanopore depends on the MC variant. As expected the percentage reduction in ionic current induced by microcystin is higher than that of acyclic peptides. ${ }^{34}$

In order to rationalize the molecular basis of specific blockage types for each MCs by the $\alpha \mathrm{HL}$ nanopore, molecular docking experiments were conducted. Such approach is the most common method employed to model the interaction between a small molecule and a protein at the atomic level, allowing the characterization of ligands behaviour in the binding site of target receptors, and generating novel information to help elucidating biological and biotechnological processes. ${ }^{36}$ Molecular docking is conducted through two steps: (1) sampling conformations of the ligand in the active site of the protein, which should be able to reproduce the experimental binding mode; and (2) ranking these conformations via a scoring function, which should also rank it highest among all generated conformations. ${ }^{37}$ From our calculations, the lowest energy docked conformations for each of the three MCs indeed fitted the region of constriction of the $\alpha \mathrm{HL}$ nanopore (Fig. 3B), in which the Arg residues in MCs would interact with Glu111 residues. Such site of interaction was also experimentally observed and/or proposed for other compounds by previous studies. $^{\text {24-30 }}$

Aiming to correlate the molecular docking results and the experimental findings, a volume exclusion assumption based on "Solvent Extractor" tool output in "Voss Volume Volexator" website was performed. ${ }^{38}$ Such approach conceit that the volume not occupied by MCs should be available to allow water molecules and ionic current. A similar approach was employed for the biological nanopore MspA, by characterizing the amount 

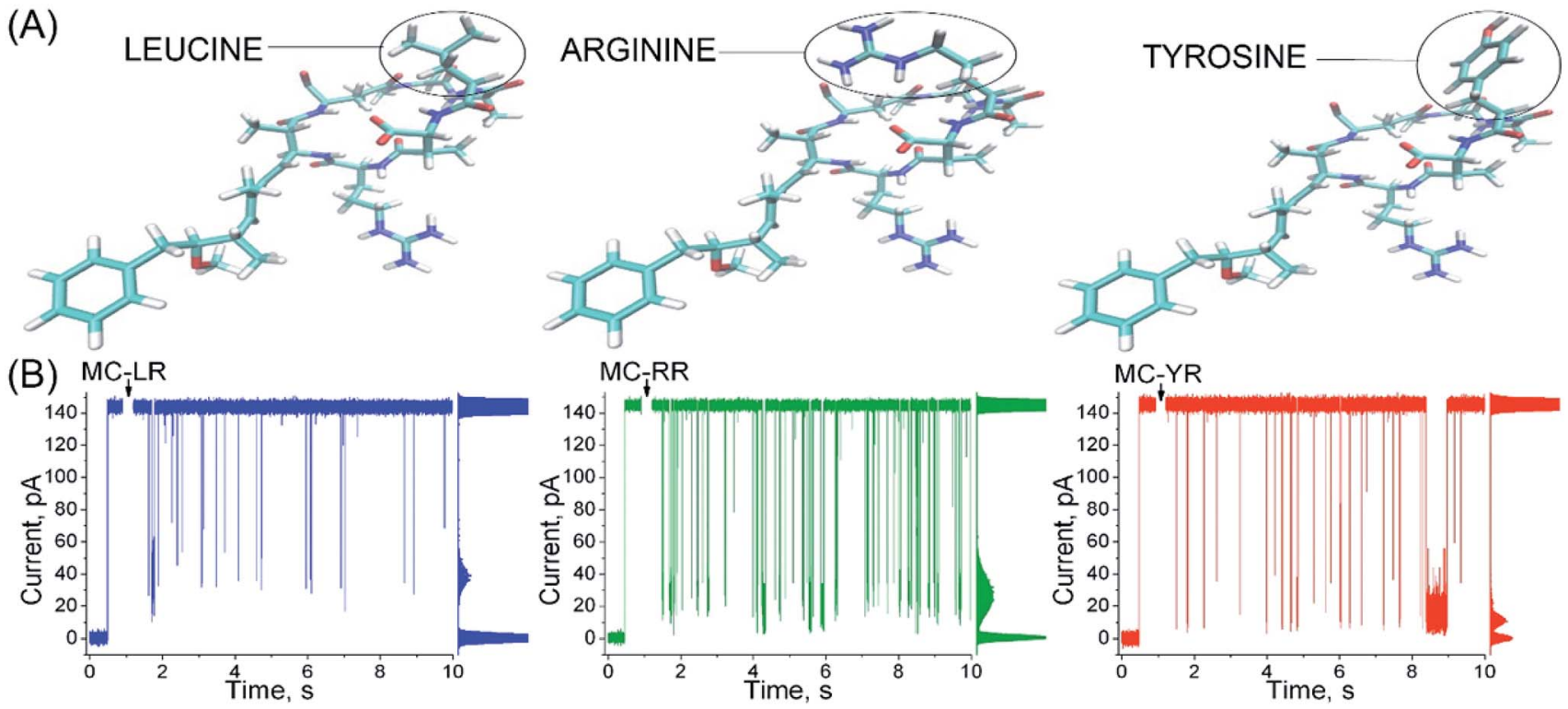

Fig. $2 \alpha$-Hemolysin nanopore detects microcystins. (A) Structural representation of variants MC-RR, MC-LR and MC-YR with residues at position $\mathrm{X} 2$ highlighted in black, (arginine), blue (leucine) and red (tyrosine) circles, respectively. (B) The profile of the microcystin-induced ionic current blockages differs to the MCs variants. Solution: $4 \mathrm{M} \mathrm{KCl}, 5 \mathrm{mM}$ Tris-HCl, (MC-RR, $1 \mu \mathrm{M}$; or MC-LR, $1 \mu \mathrm{M}$; or MC-YR, $1 \mu \mathrm{M})$, pH 7.5. Transmembrane potential, $40 \mathrm{mV}$. The all-point histograms are shown at the right of each record and can be used to give the mean value of blockage amplitudes.

Table $1 \alpha \mathrm{HL}$ nanopore constriction region volume as a function of MCs interaction and experimental values of the blocking percentage of MCs

\begin{tabular}{lcll}
\hline Nanopore state & Volume ${ }^{a}\left(\AA^{3}\right)$ & $\begin{array}{l}\text { Percentage of pore } \\
\text { obstruction (theoretical) }\end{array}$ & $\begin{array}{l}\text { Percentage of the ionic current } \\
\text { blockages amplitude (experimental) }\end{array}$ \\
\hline Unoccupied & 1113 & - & - \\
Occupied with LR & 548 & $51 \%$ & $80.1 \%$ \\
Occupied with RR & 115 & $90 \%$ & $91.6 \%$ \\
Occupied with YR & 80 & $93 \%$ & $91.6 \%$
\end{tabular}

${ }^{a}$ A total of 84 residues were considered for theoretical analysis, embracing amino acids 110 to 115 and 145 to 150 from the seven $\alpha \mathrm{HL}$ monomers.

of bulky-like water molecules in the protein constriction region, along with DNA molecules, during molecular dynamics simulations. $^{38}$ For our molecular docking poses, a quantitative correlation was observed for MC-YR in terms of percentage of pore obstruction (93\%), while both MC-RR (90\%) and MC-LR (51\%) presented the same trend (Table 1, third column). We can observe that the theoretical data are in agreement with the experimental results, as shown in more detail in the Table 1. Additionally, when three MCs variants are added simultaneously at equimolar concentrations, resulting in three blockages pattern (Fig. 3A). Amplitude of each of these three blocking patterns is like the results shown in Fig. 2B. So, $\alpha \mathrm{HL}$ nanopore can distinguish different MCs variants simultaneously present in aqueous solutions.

To analysing the dynamics of the interaction between microcystins and nanopore, we considering the process as on a simple bimolecular complexation reaction. ${ }^{29,39}$ A potential of -180 to $180 \mathrm{mV}$ was applied at increments of $20 \mathrm{mV}$ and measure the dwell time ( $\tau_{\text {off }}$ ) of the MCs variants within the $\alpha \mathrm{HL}$ nanopore (Fig. 4). For MC-RR, $\tau_{\text {off }}$ is the highest among the MCs variants at the voltage of $100 \mathrm{mV}\left(\tau_{\text {off }}=38.96 \pm 3.80 \mathrm{~ms}\right)$
(Fig. 4B). In relation to the variants LR and YR, on the potential of $-40 \mathrm{mV}$ the values of this parameters were ( $\tau_{\text {off }}=3.50 \pm 0.65$ ms) (Fig. 4A) and ( $\tau_{\text {off }}=4.54 \pm 0.63 \mathrm{~ms}$ ) (Fig. 4C), respectively.

These results show a voltage-dependent behaviour of the residence time of $\mathrm{MC}$ variants within $\alpha \mathrm{HL}$ nanopore. Analysis of the dwell times $\left(\tau_{\text {off }}\right)$ for the three MC variants has been performed at the same transmembrane potential (Fig. 5A). It indicates that the MCs variants interact differently with the nanopore. It is known that the nanopore presents the negatively charged trans region due to the presence of the amino acid lysine and aspartic acid. ${ }^{40-42}$ So, we resolved to evaluate the contribution of electrostatic forces in the interaction of microcystin with the nanopore and for this we performed calculations of electrostatic potential of these molecules. Electrostatic potential and molecular docking calculations were performed for the $\alpha \mathrm{HL}$ nanopore and MCs variants to elucidate the molecular basis of their differential interactions. The basal region on the trans side of the nanopore is rich in negatively charged residues, so, a potential of $100 \mathrm{mV}$ applied on the same side as the microcystin was added, may influence their interaction with the nanopore (Fig. 5B and C). Since the net charge of 
(A)

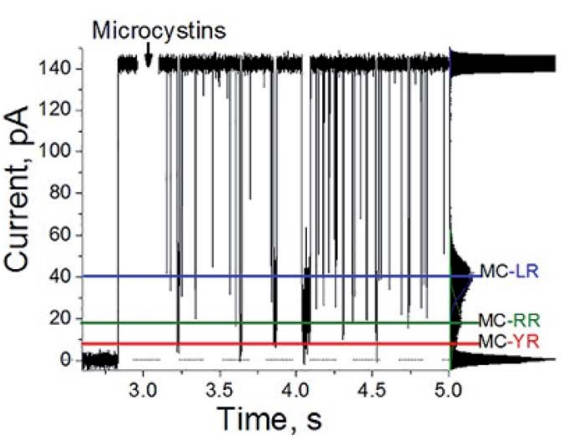

(B)

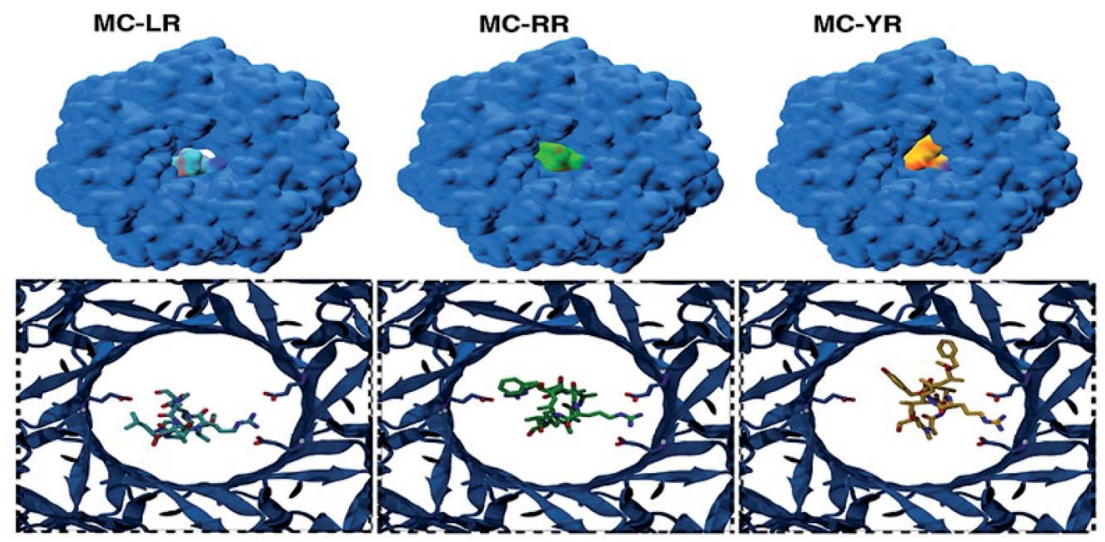

Fig. $3 \alpha$-Hemolysin nanopore is capable of discriminating MCs variants. (A) The profile of the microcystin-induced ionic current blockages for the three $\mathrm{MC}$ variant simultaneously in solution: $4 \mathrm{M} \mathrm{KCl}, 5 \mathrm{mM}$ Tris- $\mathrm{HCl}$, (MC-RR, $1 \mu \mathrm{M}$; and MC-LR, $1 \mu \mathrm{M}$; and MC-YR, $1 \mu \mathrm{M})$. The coloured lines indicate the blockage pattern for to MC-LR (blue), MC-RR (green) and MC-YR (red). (B) Docked MCs variants in the region of constriction of the $\alpha \mathrm{HL}$ nanopore. In the upper structures, the MC-nanopore complexes are represented as surface in the context of the entire protein. In the lower images, the MC-RR, MC-LR and MC-YR molecular structures are shown as they interact with Glu111 residues of the region of constriction of the $\alpha \mathrm{HL}$ nanopore.

the MC variants differs, these molecules will exhibit different interaction patterns with the nanopore, but also with the applied electrical field. In our experimental solution of $\mathrm{pH} 7.5$, the net charge of MC-RR is 0 whereas while MC-YR and MC-LR have a net charge of $-1 .^{43}$ The electrostatic potentials calculated for the two latter variants are similar though quite dissimilar from the potential obtained for MC-RR (Fig. 5D-F).

The variant MC-RR shows a mostly positive potential due to the presence of two Arg residues while the electrostatic potential generated by MC-YR and MC-LR are negative. In fact, the molecular docking poses at Fig. 3B indicate that each of the two Arg residues in MC-RR interact with two Asp127 residues in opposite sides of the nanopore, thus possibly increasing the MCs-nanopore interaction and residence time with respect to MC-YR and MC-LR. This is entirely consistent with the measurements of $\tau_{\text {off }}$ (Fig. $5 \mathrm{~A}$ ) and suggests an electrostatic interaction between MC-RR and $\alpha \mathrm{HL}$ nanopore, in agreement with the experimental data. As the inner surface (lumen) of the nanopore is positively charged, then the MC-RR, is more likely to remain longer in the entrance than in the intermediate region of the nanopore (Fig. 5C). Moreover, the predominantly negative potential surface of MC-YR and MC-LR should be less attractive to the negative potential in the trans side of the nanopore, in which their Arg residue interacts with a single Asp127 residue. As depicted in Fig. 5B, the basal region of the nanopore (trans side) is rich in aspartic acid residues, which gives a net negative charge of the trans end of the nanopore. ${ }^{40-42}$

Additionally, recent studies have shown that lithium can shield charges in base of the stem region of the $\alpha \mathrm{HL}$ nanopore. ${ }^{42}$ Based on this knowledge, we have changed the bath solution $(\mathrm{KCl} 4 \mathrm{M}$ to $\mathrm{LiCl} 4 \mathrm{M})$, in order to shielding the charges of the stem region of nanopore and verified their influence on the residence time of the MC-RR. We can observe in Fig. 5A that there was a decrease in the residence time of the MC-RR, since without the negatively charged region, the electrostatic contribution of the interaction between the MC-RR and the nanopore is lower. Furthermore, we observed a significant increase in residence time of MC-LR, so negatively charged (Fig. 5A), by
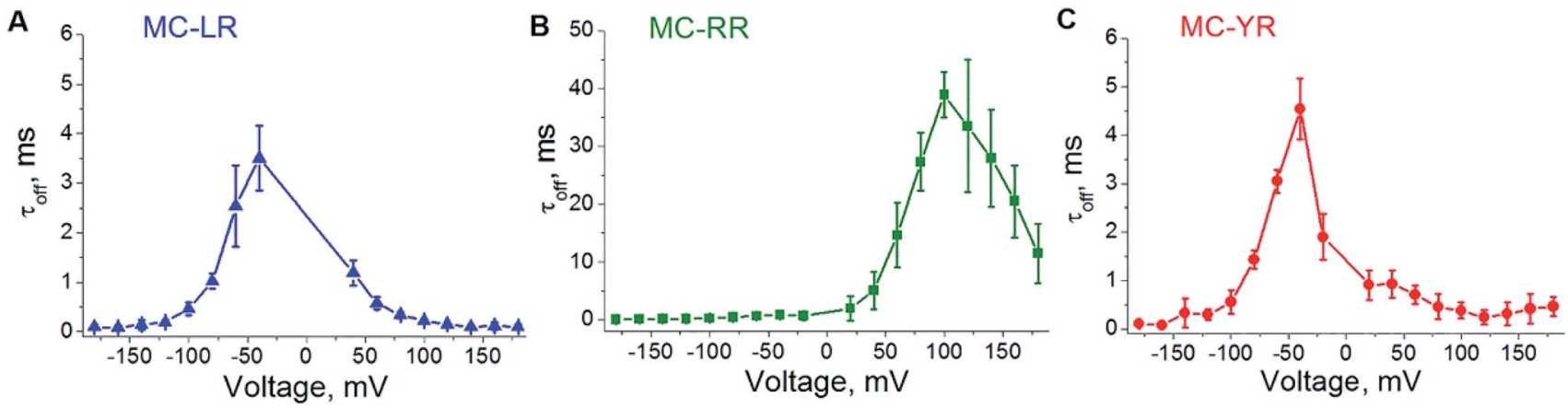

Fig. 4 Influence of the transmembrane potential in the dwell time $\left(\tau_{\text {off }}\right)$ of MCs variants within $\alpha H L$ nanopore. (A) MC-LR, (B) MC-RR, (C) MC-YR. Concentration of MCs variants is $1000 \mu \mathrm{M}$ in solution (4 M KCl, Tris- $\mathrm{HCl} 5 \mathrm{mM}, \mathrm{pH}$ 7.5). The result of at least three independent experiments (mean $\pm \mathrm{SE}$ ) is presented in each case. 


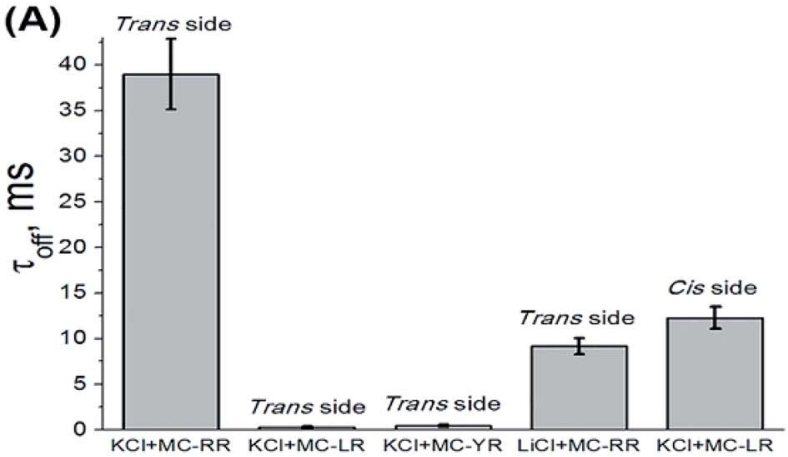

(D)

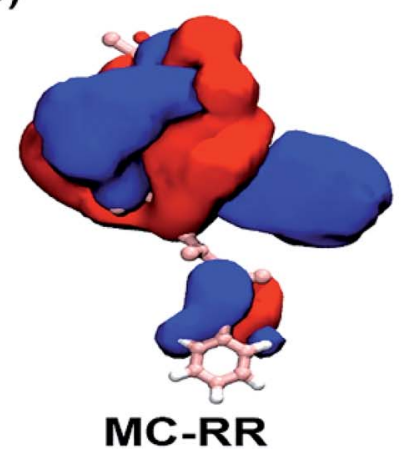

(B)

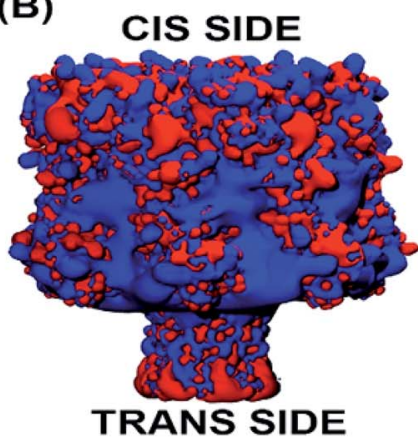

(C)

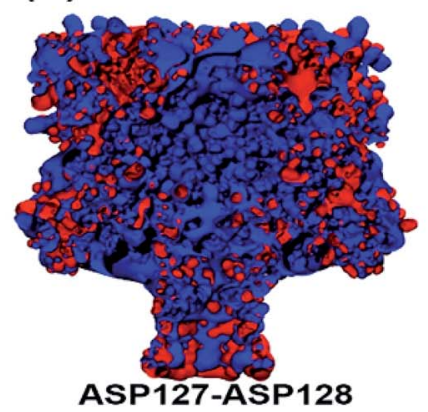

(F)

(E)

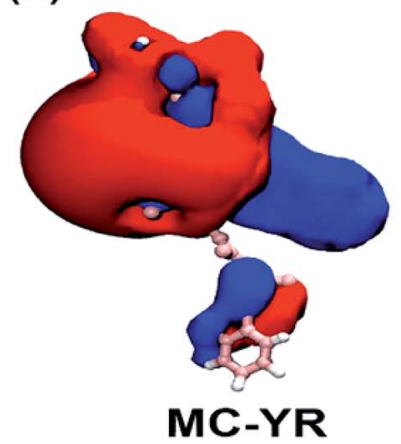

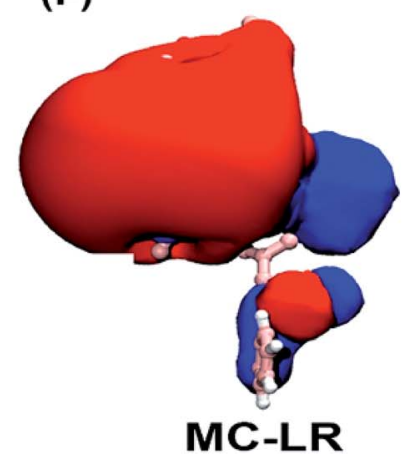

Fig. 5 Influence of the electrostatic potential in interaction of $\mathrm{MC}-\alpha \mathrm{HL}$ nanopore. (A) Residence time for $\mathrm{MC}$ variants in nanopore, solutions: $\mathrm{KCl}$ $4 \mathrm{M}$ or $\mathrm{LiCl} 4 \mathrm{M}$. The microcystin was added on the same side (cis or trans of the nanopore) in which the potential of $100 \mathrm{mV}$ was applied. (B) Electrostatic potential represented in VMD for the outer surface of the $\alpha$-HL nanopore. (C) Electrostatic potential represented in VMD for the inner surface (lumen) of the $\alpha-\mathrm{HL}$ nanopore. (D-F) Electrostatic potential represented in VMD for the MCs variants. Electrostatic potentials are shown at isosurfaces of $5 \mathrm{kTe}$ (blue) to $-5 \mathrm{kTe}$ (red).

simply changing the experimental condition, by adding the MCLR to the cis side of the nanopore. It is noteworthy that the MDgenerated structural ensembles of the MC variants are quite similar with respect to the backbone conformation. The backbone conformation is maintained due to the presence of stable intra-ring hydrogen bonds between residue pairs (D-Masp : O $\delta$ DGlu : NH, D-Masp : O $\delta$-ADDA : NH, DGlu : O-X : NH where X is either Arg, Tyr or Leu) (Fig. 1). These interactions are persistent throughout the $10 \mathrm{~ns}$ simulation time. Therefore, the calculated electrostatic potentials for the MC variants are representative of the average conformation of these cyclic peptides in solution.

Although the MC-LR and MC-YR present a similar electrostatic potential, their residence times were different (Fig. 5A), suggesting that non-electrostatic factors also influence the interaction of these toxins with the nanopore.

Aiming to elucidate the contribution of non-electrostatic factors in the interaction of microcystin with nanopore, we decided to analyse the role of hydration in the interaction of the analyte with the nanopore. In this case, we adopt the same approach of our previous studies, ${ }^{23,39}$ in which we show that the larger hydration of the analyte decreases the probability of formation and stabilization of analyte-nanopore complex. So, presence of the leucine residue gives greater hydrophobicity to MC-LR, leaving it less hydrated and increasing its residence time.

Another important parameter to analysing the kinetic process is the association rate constant $\left(k_{\mathrm{on}}\right)$. This parameter is correlated with the frequency of blockages caused by analytes. ${ }^{39,44}$ The $k_{\text {on }}$ was calculated by assuming on a simple bimolecular complexation reaction between MCs and $\alpha \mathrm{HL}$ nanopore at the single-molecule level, so that $k_{\text {on }}=1 /\left(\tau_{\text {on }} \times\right.$ $[\mathrm{MC}]$ ), where $[\mathrm{MC}]$ is the $\mathrm{MC}$ concentration and $\tau_{\mathrm{on}}$ is the characteristic time between successive blockages. ${ }^{30,39}$ The frequency of blockages of the $\alpha \mathrm{HL}$ nanopore decrease with the increasing potential for MC-LR and MC-YR, but not for MC-RR (Fig. 6). This behaviour can be expected based on the distinct net charge of the MC variants. It is known that under the solutions conditions used in our experiments ( $\mathrm{pH}$ 7.5) the MCs LR, YR and RR exhibit net charges of $-1,-1$ and 0 , respectively. ${ }^{43,45}$ However, our results indicate that MC-RR can acquire a positive charge (Fig. 6A). We suggest that this may occur because MC-RR forms complex with $\mathrm{K}^{+}$, such as occurs with some neutral molecules in the presence of $4 \mathrm{M} \mathrm{KCl}^{30,39,44}$ In this context is possible to use the nanopore as molecular tool for the rapid probing of the capabilities of different MCs variants to form complexes with different ions. One of the parameters used to evaluate the sensing capacity of the $\alpha \mathrm{HL}$ nanopore is to evaluate the behaviour of the transition rate as a function of analyte concentration. ${ }^{29,39}$ In our case, the slope of the transition rate-MC concentration increases linearly and depends on the MC variant (Fig. 6B), thus providing a basis for quantifying these cyanotoxins.

Recently method based in solid-state nanopore was used to detect MC-LR via aptamer-conjugated with gold nanoparticles and showed sensing capacity at nanomolar level. ${ }^{21}$ Our case, $\alpha \mathrm{HL}$ nanopore method detected microcystin in the micromolar 

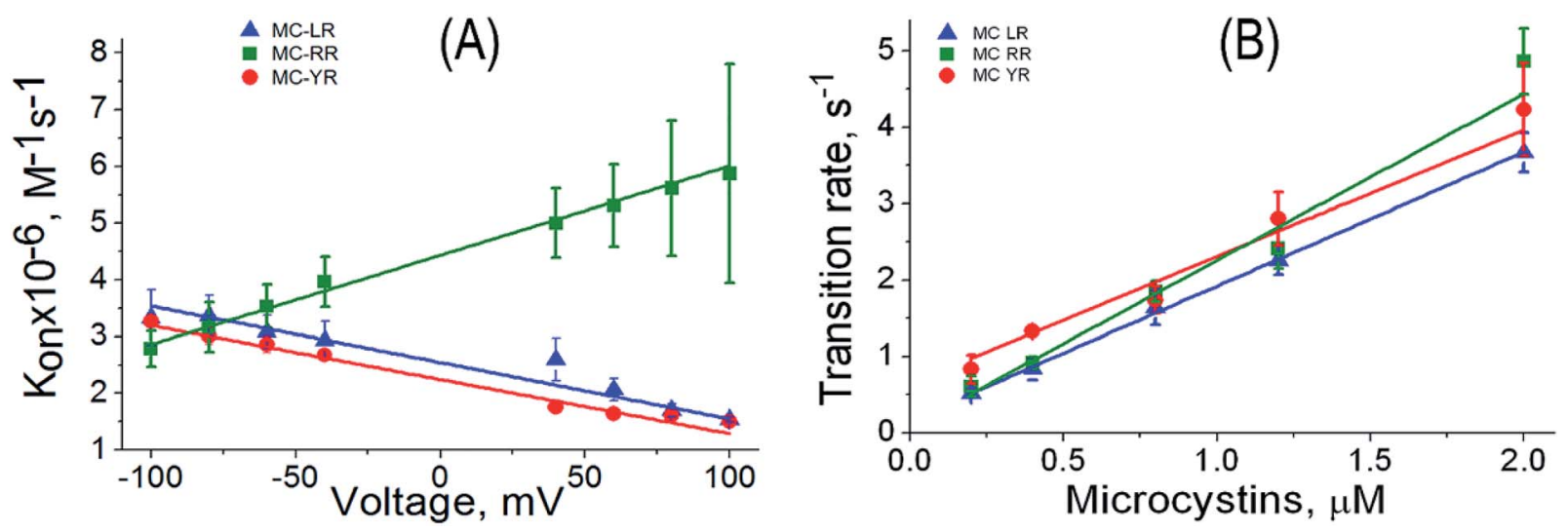

Fig. 6 (A) Influence of the transmembrane potential on the on-rate constant $\left(k_{\text {on }}\right)$ of the MC- $\alpha \mathrm{HL}$ nanopore. (B) Transition rate and MCs variants concentration. (Data points) Means of at least three separate experiments. Solution ( $4 \mathrm{M} \mathrm{KCl}$, Tris $-\mathrm{HCl} 5 \mathrm{mM}, \mathrm{pH} 7.5)$. (Data points) Means of at least three separate experiments.

range above the levels of microcystins usually found in ambient waters. On the other hand, this method does not involve labelling, modifying or conjugating microcystins with nanoparticles or antibodies, simplifying the assay procedures and reducing the time of analysis. Furthermore, several strategies, for example change in the ionic solution composition, ${ }^{29}$ may be used in future studies for improvements in the present sensing capacity of the $\alpha \mathrm{HL}$ nanopore.

\section{Experimental}

\section{Materials and methods}

Materials. All chemicals and solvents were of analyticalgrade and used as received, without further purification. High-purity water was obtained after a Milli-Q plus (Billerica, USA) treatment. The wild type of Staphylococcus aureus $\alpha$ hemolysin was purchased from Calbiochem (Madison, WI, USA). 1,2-Diphytanoyl-sn-glycero-3-phosphocholine (DPhPC) was purchased from Avanti Polar Lipids (Alabaster, AL, USA). The microcystins LR, RR and YR were purchased from Wako (Wako Pure Chemical Industries Ltd, Japan). 2-Amino-2hydroxymethyl-1,3-propanediol (TRIS) and citric acid was from Fluka (Buchs, Switzerland). KCl was purchased from SIGMA (St. Louis, MO, USA). Hexane was purchased from (J. T. Baker, Phillipsburg, NJ).

Planar lipid bilayer membranes, single channel current recording and data analysis. Solvent-free planar bilayer lipid membranes, with a capacitance of $40 \mathrm{pF}$, were formed by the lipid monolayer apposition technique, using DPhPC in hexane at $25 \pm 1{ }^{\circ} \mathrm{C}$, was described previously..$^{29}$ Because it was recently shown that the elevated $\mathrm{KCl}$ concentrations considerably improve single molecule identification by unitary protein nanopores, ${ }^{29,39}$ in this study membrane-bathing solutions contained $4 \mathrm{M} \mathrm{KCl}$ in $5 \mathrm{mM}$ Tris adjusted to $\mathrm{pH} 7.5$ with citric acid. Experiments were carried out using an Axopatch 200B amplifier (Axon Instruments, Foster City, CA) in voltage clamp mode. Membrane potential was maintained using silver/silver chloride $(\mathrm{Ag} / \mathrm{AgCl})$ electrodes in $3 \mathrm{M} \mathrm{KCl} 2 \%$ agarose bridges assembled within standard $200 \mathrm{~mL}$ pipette tips. Currents were filtered by a low-pass eight-pole Butterworth filter (model 9002; Frequency Devices, Haverhill, MA) at $15 \mathrm{kHz}$ and directly saved into the computer memory with a sampling frequency of 50 to $100 \mathrm{kHz}$. Data analysis of molecular signature parameters (mean duration and amplitude of the blockage), transition rate, and kinetic constants of the MC-nanopore interactions were done essentially as described by Rodrigues et al. ${ }^{29}$ Briefly, the on-rate constant, $k_{\mathrm{on}}$, was defined as $1 /\left(C_{\mathrm{MC}} \times \tau_{\mathrm{on}}\right)$. The characteristic time, $\tau_{\text {on }}$, was obtained from the collected time intervals between the end of one blockade event and the onset of the next. The off-rate constant, $k_{\text {off }}$, was defined as $1 / \tau_{\text {off }}$, where $\tau_{\text {off }}$ is the characteristic time of MCs staying in the pore.

Molecular dynamics (MD) simulations. Atomic coordinates of the microcystin cyclic heptapeptide were taken from the Protein DataBase (code id 1LCM) ${ }^{46}$ MD simulations were performed for microcystin MC-LR and variants where the arginine residue in position 2 was replaced by an arginine (MC-RR) or tyrosine (MC-YR). Protonation states were assigned accordingly to $\mathrm{pH}$ 7. Simulations were carried out using the GROMOS force field force parameter set 53A6 (ref. 47) in conjunction with the software package GROMACS v.4.5.4, with double precision..$^{48,49}$ The systems were placed in cubic boxes with dimension $8 \times 8 \times$ $8 \mathrm{~nm}^{3}$, treated for periodic boundary conditions, and solvated with explicit SPC model water molecules. ${ }^{50}$ The total charge of the system was neutralized by adding $\mathrm{Na}^{+}$counterions, and subsequently $\mathrm{Na}^{+}$and $\mathrm{Cl}^{-}$counterions were added to yield an ionic strength of $150 \mathrm{mM}$. Simulations were carried out in the NPT ensemble, and a time step of $2 \mathrm{fs}$ were used to integrate the equations of motion based on the Leap Frog algorithm. The temperature of the solute and solvent were separately coupled to the Berendsen thermostat at $300 \mathrm{~K}$ with a relaxation time of $0.4 \mathrm{ps}$. The pressure was maintained at $1 \mathrm{~atm}$ by isotropic coordinate scaling via the Berendsen barostat with a relaxation of 0.4 ps. $^{51}$ The bond lengths and angles were constrained by using the P-LINCS algorithm, and the geometry of the water molecules was constrained using the SETTLE algorithm, both with a tolerance of $10^{-4}$. A reaction field correction and a cutoff of $1.4 \mathrm{~nm}$ were used for both vdW and long-range electrostatic interactions with a permittivity dielectric constant of 66 . In all 
cases, the pair list for short-range non-bonded and long-range electrostatic interactions was updated with a frequency of 10 fs. Configurations of the trajectory were recorded every $0.002 \mathrm{ps}$. Atomic coordinates were visualized with the software VMD $1.92 .^{52}$

Electrostatic potential calculations. The electrostatic potential of the $\alpha \mathrm{HL}$ nanopore and the microcystin variants were calculated by solving the linearized Poisson-Boltzmann equation with the Adaptive Poisson-Boltzmann Solver package. ${ }^{53}$ The Poisson-Boltzmann equation describes the electrostatic charge $\Phi(r)$ in a medium with a dielectric scalar field $\varepsilon(r)$ and with a charge density $\rho(r)$. The solute and the solvent are represented by distinct dielectric continuums. The charge density is divided into the fixed interior charge distribution of the molecule, $\rho_{\text {int }}(r)$, and a mobile exterior charge density of the solvent and ions. The exterior is modelled as a dielectric continuum with the mobile ion density approximated by a Boltzmann distribution at temperature $T$. The linear PoissonBoltzmann equation is defined as: ${ }^{54}$

$$
\nabla \varepsilon(r) \nabla \Phi(r)=-4 \pi \rho_{\text {int }}(r)+\lambda(r) k^{2} \Phi(r)
$$

where $\varepsilon(r)$ is the dielectric constant of the solute or the solvent and $\lambda$ equals 1 for ion-accessible regions and 0 elsewhere. $\kappa^{2}$ is the modified Debye-Hückel parameter defined as:

$$
\kappa^{2}=\frac{8 \pi N_{\mathrm{A}} I}{1000 k T}
$$

where, $k$ is the Boltzmann constant, $N_{\mathrm{A}}$ is Avogadro's number and $I$ is the ionic strength of the medium. Hydrogens were added to the crystallographic coordinates with the PDB2PQR software. ${ }^{55}$ Partial charges and atomic radii were taken from the PARSE force field. ${ }^{56}$ The solute and solvent were assigned a dielectric constant of 2 and 78.5, respectively. The ionic strength of monovalent ions was set to $0.200 \mathrm{M}$ with an ion exclusion radius of $2 \AA$ and a temperature of $298 \mathrm{~K}$. The finitedifference Poisson-Boltzmann calculations employed grids with $257 \times 257 \times 257(\alpha \mathrm{HL})$ and $33 \times 33 \times 65(\mathrm{MCs})$ points in $x$, $y, z$ directions, respectively.

Molecular docking studies. Atomics coordinates for the $\alpha \mathrm{HL}$ heptameric channel was retrieved from PDB ID 7AHL, ${ }^{57}$ while representative structures from the microcystins were retrieved from MD simulations. The molecular dockings were performed in two protein regions, (1) the constriction and (2) the "trans" pore exit, using Dockthor online portal ${ }^{58}$ with the standard program docking parameters. Briefly, its built-in genetic algorithm was employed in a total of 30 runs, each one with an initial population of 1000 random individuals, and a maximum number of $1.0 \times 10^{7}$ energy evaluations. The atomic coordinates of the lowest energy conformations were clustered based in a root-mean-squared-deviation (RMSD) of $2.0 \AA$. The output conformers for the three MCs, which showed favourable interaction energies with $\alpha \mathrm{HL}$, were sorted in order of increasing energy, and the lowest energy docked conformations were retrieved for further analysis. The volume analyses were performed using the "Solvent Extractor" tool in "Voss Volume Volexator" website, using the standard program parameters. ${ }^{59}$

\section{Conclusions}

In summary, we demonstrate that the $\alpha \mathrm{HL}$ nanopore can detect and discriminate three structural variants (LR, YR, RR) of microcystins simultaneously, by the difference in the blocking profiles in the ionic current induced by each of the variants. Using computational methods, it was found that electrostatic interactions contribute to the interaction between MCs and the base of the stem region nanopore. The positively charged profile acquired by MC-RR facilitates their interaction with the nanopore, which does not occur with the MCs LR and YR. In addition, being less hydrated, MC-LR has more interaction with the nanopore than the MC-YR. Finally, we concluded that $\alpha \mathrm{HL}$ nanopore discriminates variants of microcystins by a mechanism based mainly on electrostatic interaction. Therefore, we propose the use of the nanopore-based technology as an auxiliary method of MCs analysis in aqueous solutions.

\section{Conflicts of interest}

There are no conflicts to declare.

\section{Acknowledgements}

This work was supported by the Conselho Nacional de Desenvolvimento Científico e Tecnológico (CNPq), Grant 470008/ 2012-1 and, Fundação de Amparo à Ciência e Tecnologia do Estado de Pernambuco (FACEPE), Grant APQ-0707-2.09/15, Brazil.

\section{Notes and references}

1 J. Fawell, R. Mitchell, D. Everett and R. Hill, The toxicity of cyanobacterial toxins in the mouse: I Microcystin-LR, Hum. Exp. Toxicol., 1999, 18, 162-167.

2 A. Srivastava, S. Singh, C. Y. Ahn, H. M. Oh and R. K. Asthana, Monitoring approaches for a toxic cyanobacterial bloom, Environ. Sci. Technol., 2013, 47(16), 8999-9013.

$3 \mathrm{~J}$. McElhiney and L. A. Lawton, Detection of the cyanobacterial hepatotoxins microcystins, Toxicol. Appl. Pharmacol., 2005, 203(3), 219-230.

4 J. Puddick, M. R. Prinsep, S. A. Wood, S. A. F. Kaufononga, S. C. Cary and D. P. Hamilton, High levels of structural diversity observed in microcystins from microcystis CAWBG11 and characterization of six new microcystin congeners, Mar. Drugs, 2014, 12(11), 5372-5395.

5 W. W. Carmichael, S. M. Azevedo, J. S. An, R. J. Molica, E. M. Jochimsen, S. Lau, K. L. Rinehart, G. R. Shaw and G. K. Eaglesham, Human Fatalities from Cyanobacteria: Chemical and Biological Evidence for Cyanotoxins, Environ. Health Perspect., 2001, 109(7), 663-668.

6 D. Drobac, N. Tokodi, J. Simeunović, V. Baltić, D. Stanić and Z. Svirčev, Human exposure to cyanotoxins and their effects on health, Arh. Hig. Rada Toksikol., 2013, 64(2), 305-316.

7 S. Pouria, A. De Andrade, J. Barbosa, R. L. Cavalcanti, V. T. S. Barreto, C. J. Ward, W. Preiser, G. K. Poon, G. H. Neild and G. A. Codd, Fatal microcystin intoxication 
in haemodialysis unit in Caruaru, Brazil, Lancet, 1998, 352(9121), 21-26.

8 R. M. Dawson, The toxicology of microcystins, Toxicon, 1998, 36(7), 953-962.

9 M. M. Gehringer, Microcystin-LR and okadaic acid-induced cellular effects: A dualistic response, FEBS Lett., 2004, 557, 1-8.

10 H. G. Gorchev and G. Ozolins, Guidelines for Drinking-water Quality, WHO, 2011, vol. 1, p. 564.

11 H. W. Paerl and J. Huisman, Blooms like it hot, Science, 2008, 320(5872), 57-58.

12 H. W. Paerl, Mitigating Harmful Cyanobacterial Blooms in a Human- and Climatically-Impacted World, Life, 2014, 4(4), 988-1012.

13 C. Butterwick, S. I. Heaney and J. F. Talling, Diversity in the influence of temperature on the growth rates of freshwater algae, and its ecological relevance, Freshwater Biol., 2005, 50(2), 291-300.

14 A. E. Poste, R. E. Hecky and S. J. Guildford, Evaluating microcystin exposure risk through fish consumption, Environ. Sci. Technol., 2011, 45(13), 5806-5811.

15 S. Singh, A. Srivastava, H. M. Oh, C. Y. Ahn, G. G. Choi and R. K. Asthana, Recent trends in development of biosensors for detection of microcystin, Toxicon, 2012, 60(5), 878-894.

16 L. M. Alvarenga, J. Muzard, A. Ledreux, C. Bernard and P. Billiald, Colorimetric engineered immunoprobe for the detection and quantification of microcystins, J. Immunol. Methods, 2014, 406, 124-130.

17 J. McElhiney, L. A. Lawton and A. J. R. Porter, Detection and quantification of microcystins (cyanobacterial hepatotoxins) with recombinant antibody fragments isolated from a naive human phage display library, FEMS Microbiol. Lett., 2000, 193, 83-88.

18 Nanopore-based Technology, ed. M. E. Gracheva, Humana Press, New York, 2012.

19 J. P. Aguiar, J. J. S. Júnior, D. C. Machado, M. C. A. Melo and C. G. Rodrigues, Biossensoriamento estocástico via nanoporo proteico individual no desenvolvimento de ferramentas analíticas, Quim. Nova, 2015, 38(6), 817-827.

20 L. Q. Gu, O. Braha, S. Conlan, S. Cheley and H. Bayley, Stochastic sensing of organic analytes by a pore-forming protein containing a molecular adapter, Nature, 1999, 398(6729), 686-690.

21 F. He, L. Liang, S. Zhou, W. Xie, S. He, Y. Wang, C. Tlili, S. Tong and D. Wang, Label-Free Sensitive Detection of Microcystin-LR via Aptamer-Conjugated Gold Nanoparticles Based on Solid-State Nanopores, Langmuir, 2018, 34(49), 14825-14833.

22 P. G. Merzlyak, L. N. Yuldasheva, C. G. Rodrigues, C. M. Carneiro, O. V. Krasilnikov and S. M. Bezrukov, Polymeric nonelectrolytes to probe pore geometry: application to the alpha-toxin transmembrane channel, Biophys. J., 1999, 77(6), 3023-3033.

23 P. A. Gurnev and E. M. Nestorovich, Channel-forming bacterial toxins in biosensing and macromolecule delivery, Toxins, 2014, 6(8), 2483-2540.
24 J. W. F. Robertson, C. G. Rodrigues, V. M. Stanford, K. A. Rubinson, O. V. Krasilnikov and J. J. Kasianowicz, Single-molecule mass spectrometry in solution using a solitary nanopore, Proc. Natl. Acad. Sci. U. S. A., 2007, 104(20), 8207-8211.

$25 \mathrm{H}$. Bayley, Nanopore sequencing: From imagination to reality, Clin. Chem., 2015, 61(1), 25-31.

26 X. F. Kang, S. Cheley, X. Guan and H. Bayley, Stochastic detection of enantiomers, J. Am. Chem. Soc., 2006, 128(33), 10684-10685.

27 R. Kawano, T. Osaki, H. Sasaki, M. Takinoue, S. Yoshizawa and S. Takeuchi, Rapid detection of a cocaine-binding aptamer using biological nanopores on a chip, J. Am. Chem. Soc., 2011, 133(22), 8474-8477.

28 G. Baaken, I. Halimeh, L. Bacri, J. Pelta, A. Oukhaled and J. C. Behrends, High-Resolution Size-Discrimination of Single Nonionic Synthetic Polymers with a Highly Charged Biological Nanopore, ACS Nano, 2015, 9(6), 6443-6449.

29 C. G. Rodrigues, D. C. Machado, A. M. B. Silva, J. J. S. Júnior and O. V. Krasilnikov, Hofmeister effect in confined spaces: Halogen ions and single molecule detection, Biophys. J., 2011, 100(12), 2929-2935.

30 C. G. Rodrigues, D. C. Machado, S. F. Chevtchenko and O. V. Krasilnikov, Mechanism of $\mathrm{KCl}$ enhancement in detection of nonionic polymers by nanopore sensors, Biophys. J., 2008, 95(11), 5186-5192.

31 S. M. Bezrukov, Ion channels as molecular coulter counters to probe metabolite transport, J. Membr. Biol., 2000, 174(1), 1-13.

32 D. Branton, D. W. Deamer, A. Marziali, H. Bayley, S. A. Benner, T. Butler, M. Di Ventra, S. Garaj, A. Hibbs, X. Huang, S. B. Jovanovich, P. S. Krstic, S. Lindsay, X. S. Ling, C. H. Mastrangelo, A. Meller, J. S. Oliver, Y. V. Pershin, J. M. Ramsey, R. Riehn, G. V. Soni, V. Tabard-Cossa, M. Wanunu, M. Wiggin and J. Schloss, The potential and challenges of nanopore sequencing, Nat. Biotechnol., 2008, 26(10), 1146-1153.

33 L. Q. Gu, M. Wanunu, M. X. Wang, L. McReynolds and Y. Wang, Detection of miRNAs with a nanopore singlemolecule counter, Expert Rev. Mol. Diagn., 2012, 12(6), 573584.

34 L. Movileanu, J. P. Schmittschmitt, J. M. Scholtz and H. Bayley, Interactions of peptides with a protein pore, Biophys. J., 2005, 89(2), 1030-1045.

35 Q. Zhao, D. A. Jayawardhana, D. Wang and X. Guan, Study of peptide transport through engineered protein channels, $J$. Phys. Chem. B, 2009, 113(11), 3572-3578.

36 B. McConkey, V. Sobolev and M. Edelman, The Performance of Current Methods in Ligand-Protein Docking, Curr. Sci., 2002, 83(7), 845-856.

37 X.-Y. Meng, H.-X. Zhang, M. Mezei and M. Cui, Molecular Docking: A powerful approach for structure-based drug discovery, Curr. Comput.-Aided Drug Des., 2011, 7(2), 146157.

38 S. Bhattacharya, J. Yoo and A. Aksimentiev, Water Mediates Recognition of DNA Sequence via Ionic Current Blockade in a Biological Nanopore, ACS Nano, 2016, 10(4), 4644-4651. 
39 D. C. Machado, J. J. S. Júnior, M. C. A. Melo, A. M. B. Silva, A. Fontes and C. G. Rodrigues, Effects of alkali and ammonium ions in the detection of poly(ethyleneglycol) by alpha-hemolysin nanopore sensor, $R S C A d v$., 2016, 6(61), 56647-56655.

40 S. Y. Noskov, W. Im and B. Roux, Ion Permeation through the a-Hemolysin Channel: Theoretical Studies Based on Brownian Dynamics and Poisson-Nernst-Plank Electrodiffusion Theory, Biophys. J., 2004, 87, 2299-2309.

41 L. R. Teixeira, P. G. Merzlyak, A. Valeva and O. V. Krasilnikov, Interaction of heparins and dextran sulfates with a mesoscopic protein nanopore, Biophys. J., 2009, 97(11), 2894-2903.

42 S. Bhattacharya, J. Muzard, L. Payet, J. Math, U. Bockelmann, A. Aksimentiev and V. Viasnoff, Rectification of the Current in $\alpha$-Hemolysin Pore Depends on the Cation Type: The Alkali Series Probed by Molecular Dynamics Simulations and Experiments, J. Phys. Chem., 2011, 115(10), 4255-4264.

43 P. G. J. Maagd, A. J. Hendriks, W. Seinena and D. T. H. M. Sijma, pH-Dependent hydrophobicity of the cyanobacteria toxin microcystin-LR, Water Res., 1999, 33(3), 677-680.

44 M. F. Breton, F. Discala, L. Bacri, D. Foster, J. Pelta and A. Oulchaled, Exploration of Neutral Versus Polyelectrolyte Behavior of Poly(ethylene glycol)s in Alkali Ion Solutions using Single-Nanopore Recording, J. Phys. Chem. Lett., 2013, 4(13), 2202-2208.

45 I. Liu, L. A. Lawton and P. K. Robertson, Mechanistic studies of the photocatalytic oxidation of microcystin-LR: an investigation of byproducts of the decomposition process, Environ. Sci. Technol., 2003, 37(14), 3214-3219.

46 G. B. Trogen, A. Annila, J. Eriksson, M. Kontteli, J. Meriluoto, I. Sethson, J. Zdunek and U. Edlund, Conformational studies of microcystin-LR using NMR spectroscopy and molecular dynamics calculations, Biochemistry, 1996, 35(10), 31973205.

47 C. Oostenbrink, T. A. Soares, N. F. A. Van Der Vegt and W. F. Van Gunsteren, Validation of the 53A6 GROMOS force field, Eur. Biophys. J., 2005, 34(4), 273-284.

48 D. Van Der Spoel, E. Lindahl, B. Hess, G. Groenhof, A. E. Mark and H. J. C. Berendsen, GROMACS: Fast, flexible, and free, J. Comput. Chem., 2005, 26(16), 1701-1718.
49 B. Hess, C. Kutzner, D. Van Der Spoel and E. Lindahl, GROMACS 4: Algorithms for highly efficient, loadbalanced, and scalable molecular simulation, J. Chem. Theory Comput., 2008, 4(3), 435-447.

50 H. J. C. Berendsen, J. P. M. Postma, W. F. van Gunsteren and J. Hermans, Interaction models for water in relation to protein hydration, in Intermolecular Forces, ed. B. Pullman, Springer Netherlands, 1981, pp. 331-342.

51 H. J. C. Berendsen, J. P. M. Postma, W. F. van Gunsteren, A. DiNola and J. R. Haak, Molecular dynamics with coupling to an external bath, J. Chem. Phys., 1984, 81(8), 3684-3690.

52 W. Humphrey, A. Dalke and K. Schulten, VMD: Visual molecular dynamics, J. Mol. Graphics, 1996, 14(1), 33-38.

53 N. A. Baker, D. Sept, S. Joseph, M. J. Holst and J. A. McCammon, Electrostatics of nanosystems: application to microtubules and the ribosome, Proc. Natl. Acad. Sci. U. S. A., 2001, 98(18), 10037-10041.

54 F. Fogolari, A. Brigo and H. Molinari, The PoissonBoltzmann equation for biomolecular electrostatics: A tool for structural biology, J. Mol. Recognit., 2002, 15(6), 377-392.

55 T. J. Dolinsky, J. E. Nielsen, J. A. McCammon and N. A. Baker, PDB2PQR: An automated pipeline for the setup of PoissonBoltzmann electrostatics calculations, Nucleic Acids Res., 2004, 32, 665-667.

56 D. Sitkoff, K. A. Sharp and B. Honig, Accurate Calculation of Hydration Free Energies Using Macroscopic Solvent Models, J. Phys. Chem., 1994, 98(7), 1978-1988.

57 L. Song, M. R. Hobaugh, C. Shustak, S. Cheley, H. Bayley and J. E. Gouaux, Structure of staphylococcal alpha-hemolysin, a heptameric transmembrane pore, Science, 1996, 274(5294), 1859-1865.

58 C. S. Magalhães, D. M. Almeida, H. J. C. Barbosa and L. E. Dardenne, A dynamic niching genetic algorithm strategy for docking highly flexible ligands, Inf. Sci., 2014, 289, 206-224.

59 N. R. Voss and M. Gerstein, 3V: Cavity, channel and cleft volume calculator and extractor, Nucleic Acids Res., 2010, 38, 555-562. 